\title{
Correction to: Differences in gut microbiota associated with age, sex, and stool consistency in healthy Japanese subjects
}

\author{
Tomohisa Takagi $^{1,2} \cdot$ Yuji Naito $^{1} \cdot$ Ryo Inoue $^{3} \cdot$ Saori Kashiwagi $^{1}$. \\ Kazuhiko Uchiyama $^{1}$ Katsura Mizushima ${ }^{1} \cdot$ Saeko Tsuchiya $^{1} \cdot$ Osamu Dohi $^{1}$. \\ Naohisa Yoshida ${ }^{1} \cdot$ Kazuhiro Kamada $^{1}$. Takeshi Ishikawa ${ }^{1} \cdot$ Osamu Handa $^{1}$ • \\ Hideyuki Konishi ${ }^{1} \cdot$ Kayo Okuda $^{4} \cdot$ Yoshimasa Tsujimoto $^{4} \cdot$ Hiromu Ohnogi $^{4}$. \\ Yoshito Itoh ${ }^{1}$
}

Published online: 6 September 2018

(C) Japanese Society of Gastroenterology 2018

\section{Correction to: J Gastroenterol https://doi.org/10.1007/s00535-018-1488-5}

The authors would like to correct the errors in the publication of the original article. The correction details are given below for your reading.

The first sentence in the second paragraph beneath the heading "Microbiome analysis" in third page should be "The observed species, Chaol and Shannon phylogenetic diversity indices were calculated by the R "phyloseq" package and were statistically analyzed using a Wilcoxon rank-sum test."

The last two sentences in the first paragraph, in fifth page should be "In the younger group (20-39 years), the relative abundance of Bifidobacterium $(p<0.01)$ and $R u$ minococcus $(p<0.05)$ substantially increased in female subjects, and the relative abundance of Escherichia $(p<0.05)$ significantly increased in male subjects. In the middle age group (40-59 years), the relative abundance of Prevotella $(p<0.05)$ and Megamonas $(p<0.05)$

The original article can be found online at https://doi.org/10.1007/ s00535-018-1488-5.

Tomohisa Takagi

takatomo@koto.kpu-m.ac.jp

1 Molecular Gastroenterology and Hepatology, Graduate School of Medical Science, Kyoto Prefectural University of Medicine, 465 Kajii-cho, Kawaramachi-Hirokoji, Kamigyoku, Kyoto 602-8566, Japan

2 Department for Medical Innovation and Translational Medical Science, Graduate School of Medical Science, Kyoto Prefectural University of Medicine, Kyoto 602-8566, Japan

3 Laboratory of Animal Science, Kyoto Prefectural University, Kyoto 606-8522, Japan

4 Takara Bio Inc., Shiga 525-0058, Japan increased in male subjects, and the relative abundance of Ruminococcus $(p<0.01)$ and Oscillospira $(p<0.01)$ significantly increased in female subjects. In the elderly group (60 years or older), the relative abundance of Bacteroides $(p<0.01)$ and Turicibacter $(p<0.05)$ increased in female subjects, and the relative abundance of Prevotella $(p<0.01)$, Megasphaera $(p<0.05)$ significantly increased in male subjects (Supplementary Fig. 4)."

The third sentence of the fourth paragraph, in seventh page should be "Characteristically, the genera Prevotella (middle age group and elderly group) and Megamonas (middle age group) were notably increased in male subjects, and the genera Bifidobacterium (younger group) was strikingly increased in female subjects".

In addition, Figs. 4 and 8 were published incorrectly. The correct Figs. 4 and 8 are given in the following pages: 


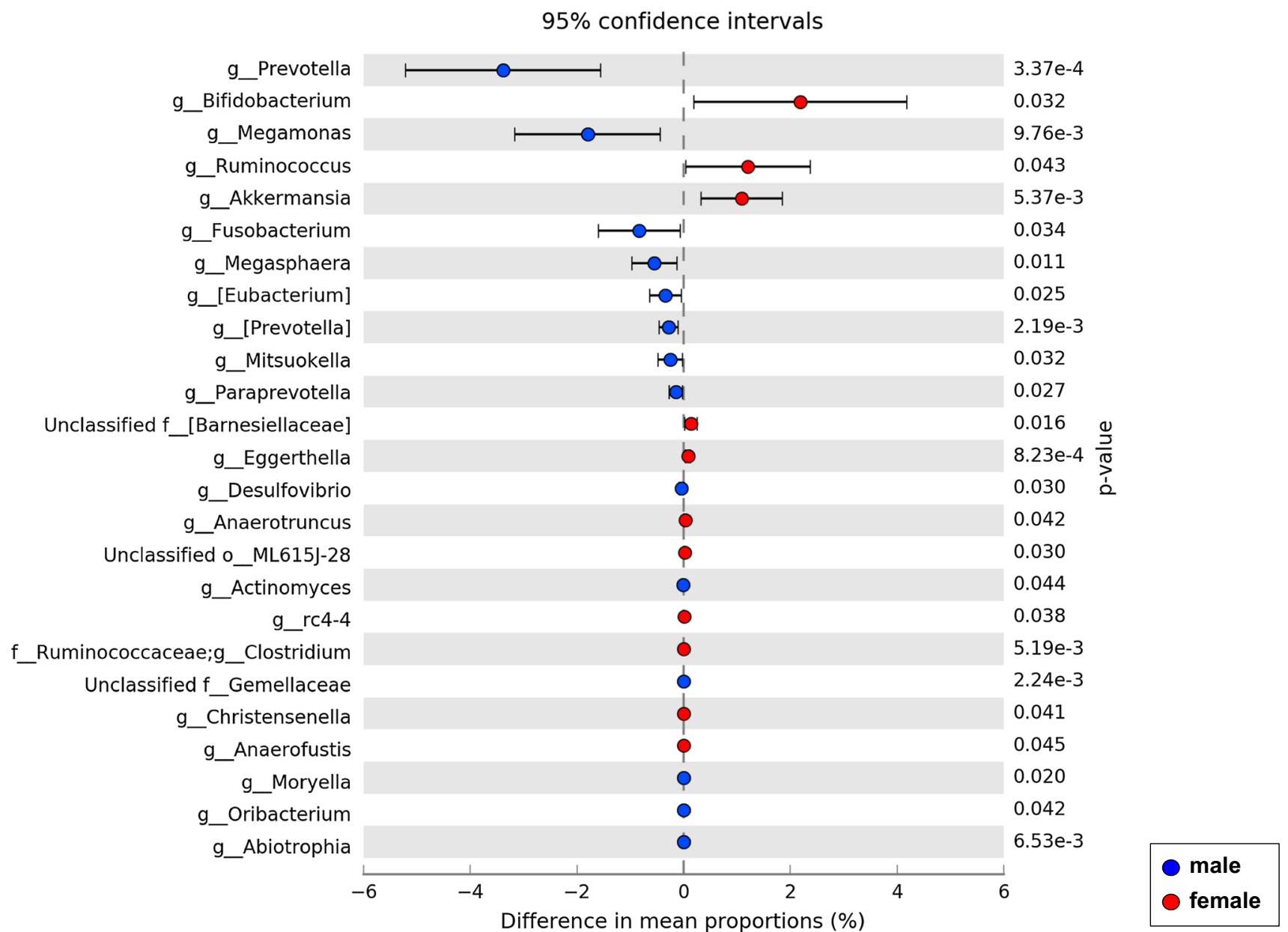

Fig. 4 The relative abundance of gut microbiota in male and female subjects. The genera were significantly different between male and female subjects 


\section{(A) Male}

(a) Bilophila

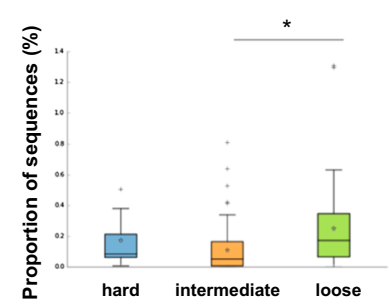

\section{(B) Female}

(a) Adlercreutzia

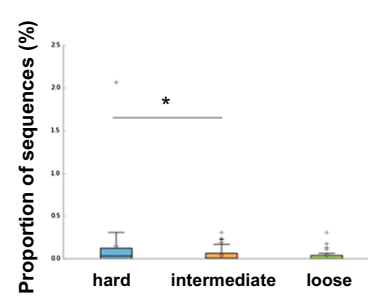

(e) Haemophilus

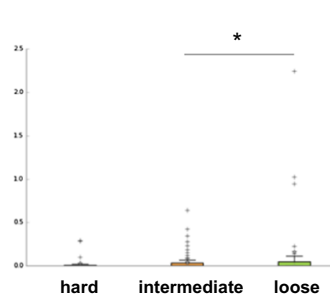

(b) Fusobacterium

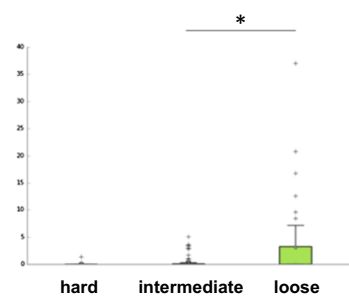

(b) Bifidobacterium

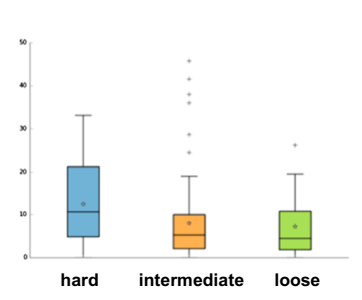

(f) Serratia

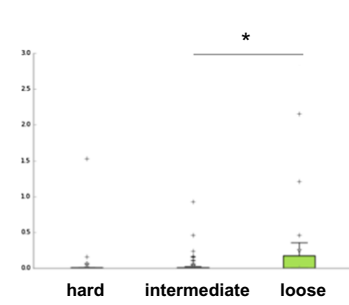

(c) Oscillospira

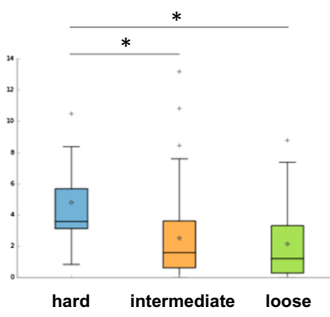

(d) Unclassified f Ruminococcacease

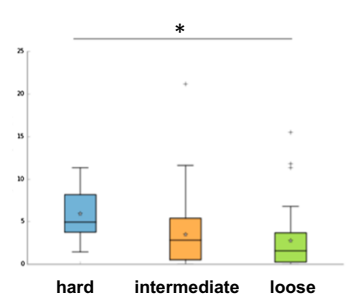

(d) Dorea

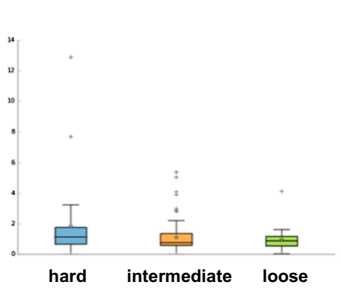

\section{(f) Turicibacter}
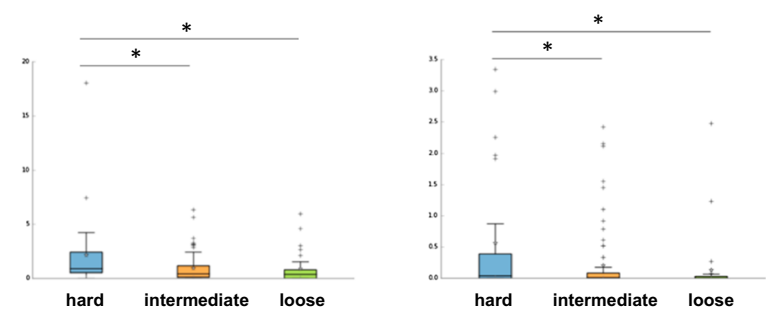

Fig. 8 Comparative analyses of the microbial community at the genus level for each stool consistency group. Genera were significantly different among each stool consistency group for male and female subjects. Stool consistency was categorized into three groups: hard (BSS 1 or 2), intermediate (BSS 3 or 4), and loose (BSS 5 or 6) 